\title{
APPROXIMATION OF SHEAVES ON ALGEBRAIC STACKS
}

\author{
DAVID RYDH
}

\begin{abstract}
Raynaud-Gruson characterized flat and pure morphisms between affine schemes in terms of projective modules. We give a similar characterization for non-affine morphisms. As an application, we show that every quasi-coherent sheaf is the union of its finitely generated quasi-coherent subsheaves on any quasi-compact and quasi-separated algebraic stack.
\end{abstract}

\section{INTRODUCTION}

It is well-known that on a noetherian scheme every quasi-coherent sheaf is the union of its coherent subsheaves [EGAIa, Cor. 9.4.9]. This is also true for noetherian algebraic stacks [LMB00, Prop. 15.4].

For a non-noetherian scheme or algebraic stack $X$, this question splits up into two questions.

(i) Is every quasi-coherent $\mathcal{O}_{X}$-module the union of its quasi-coherent submodules of finite type?

(ii) Is every quasi-coherent $\mathcal{O}_{X}$-module a directed colimit of finitely presented $\mathcal{O}_{X}$-modules?

When these questions have positive answers, we say that $X$ has the partial completeness property and completeness property respectively. The second property implies the first (take images).

It is known that quasi-compact and quasi-separated schemes have the completeness property $\mathrm{EGA}_{\mathrm{I}} \mathrm{b}$, §6.9]. In Ryd15, Thm. A] it was shown that many stacks, including quasi-compact and quasi-separated algebraic spaces and Deligne-Mumford stacks, have the completeness property. With current technology, this result only applies to relatively few algebraic stacks with infinite stabilizer groups.

The main result of this paper settles the partial completeness property for every reasonable stack.

Theorem. Let $X$ be a quasi-compact and quasi-separated algebraic stack. Then every quasi-coherent $\mathcal{O}_{X}$-module is the union of its quasi-coherent submodules of finite type.

An important application of the theorem is that when $X$ in addition has affine stabilizer groups, then there exists a finitely presented filtration of $X$ with strata that are global quotient stacks [HR14a, Prop. 2.6 (i)].

Date: 2015-05-07.

2010 Mathematics Subject Classification. Primary 14A20.

Key words and phrases. Noetherian approximation, pure, projective, algebraic stacks.

Supported by the Swedish Research Council grant no 2011-5599. 
This is used to obtain a criterion for an algebraic stack to have finite cohomological dimension [HR14a, Thm. 2.1] and to extend Tannaka duality to non-noetherian stacks [HR14b, Thm. 1.4].

The key idea in the proof of the main theorem is to use projective modules instead of flat modules. The main lemma (6.2) on existence of minimal modules goes back to Serre $\left[\mathrm{SGA}_{3}\right.$, Exp. VIB, 11.8, 11.10.1] in the context of coalgebras and comodules. Here projectivity cannot be replaced with flatness. The bulk of the paper extends this result to non-affine pure morphisms (Theorem 6.3). For this, we use a new characterization of pure morphisms between stacks in terms of projectivity (Theorem 5.3). This generalizes the characterization of affine pure morphisms due to Raynaud-Gruson [RG71, Thm. I.3.3.5].

The main result naturally leads to the following conjectures.

Conjecture A. If $X$ is a quasi-compact and quasi-separated algebraic stack, then $X$ has the completeness property.

Conjecture B. If $X$ is a quasi-compact and quasi-separated algebraic stack, then $X$ has an approximation, that is, there exists a factorization $X \rightarrow$ $X_{0} \rightarrow$ Spec $\mathbb{Z}$ where $X \rightarrow X_{0}$ is affine and $X_{0}$ is of finite presentation over $\operatorname{Spec} \mathbb{Z}$.

The second conjecture implies the first conjecture. Our proof of the main theorem first reduces the question to the case when there is a pure presentation. The conjectures can also be reduced to this seemingly simpler situation (see Remark 7.6).

In Sections 24, we recall and extend some notions from schemes to algebraic stacks. This includes (1) locally free and locally projective modules, (2) assassins and schematically dominant morphisms, and (3) pure morphisms. In Section 5, we give a characterization of pure morphisms in terms of projectivity (Theorem 5.3). In Section [6, we prove the existence of minimal subsheaves for pure morphisms. In Section 7, we prove the main theorem. In the last section, we give some applications to the main theorem.

We follow the terminology of $[\mathrm{SP}]$ and do not impose any separation conditions on a general algebraic stack. An algebraic stack is quasi-separated if its diagonal is quasi-compact and quasi-separated, that is, if the diagonal and the double diagonal are quasi-compact.

1.1. Acknowledgments. It is my pleasure to acknowledge useful discussions with Jack Hall and useful comments from Martin Brandenburg and Matthieu Romagny. I would also like to express my gratitude to the referees for their many useful suggestions and corrections that improved the paper.

\section{LOCALLY FREE AND LOCALLY PROJECTIVE MODULES}

In this section, we recall some standard results on infinitely generated projective modules due to Kaplansky, Bass and Raynaud-Gruson.

Definition (2.1). Let $X$ be an algebraic stack. We say that a quasicoherent sheaf $\mathcal{F}$ is locally free (resp. locally projective) if there exists a 
jointly surjective family of flat morphisms $p_{i}$ : Spec $A_{i} \rightarrow X$, locally of finite presentation, such that $p_{i}^{*} \mathcal{F}$ is free (resp. projective) for every $i$.

We do not require that $p_{i}^{*} \mathcal{F}$ has finite rank in the definition of locally free. Note that the properties locally free and locally projective are stable under arbitrary pull-back and are local for the fppf-topology. We have the implications: locally free $\Longrightarrow$ locally projective $\Longrightarrow$ flat.

If $x \in|X|$ is a point, then we define the $\operatorname{rank} \operatorname{rk}_{\mathcal{F}}(x)$ of $\mathcal{F}$ at $x$ as the cardinality of a basis of the $k$-vector space $\varphi^{*} \mathcal{F}$ for any representative $\varphi$ : Spec $k \rightarrow X$ of $x$. Since flat morphisms that are locally of finite presentation are open, the rank of $\mathcal{F}$ is locally constant on $|X|$ if $\mathcal{F}$ is locally free.

The rank does not behave so well for flat modules that are not finitely generated. If $A=\mathbb{Z}$ and $M=\mathbb{Q}$, then the rank of $M$ is not upper semicontinuous. The rank of projective modules is more well-behaved.

Lemma (2.2) (Kaplansky Kap58]). If $A$ is a local ring, then every projective A-module is free.

Thus, if $X$ is a quasi-separated 1 algebraic stack and $\mathcal{F}$ is a locally projective $\mathcal{O}_{X}$-module, then

(i) the rank of $\mathcal{F}$ is constant on irreducible components of $X$; and

(ii) if $X$ has a finite number of irreducible components (e.g., $X$ noetherian), then the rank is locally constant.

Nevertheless, even if $M$ is projective and has finite rank at every point, the rank need not be locally constant. Bass gives an example, due to Kaplansky, of a projective module of rank $\leq 1$ such that the locus where the module has rank 0 is closed but not open [Bas63, p. 31, (2)]. We now give a similar example.

Example (2.3). Let $k$ be an algebraically closed field and let $A=T(k[x])$ be the absolutely flat ring associated to the polynomial ring $k[x]$ [Oli68, Prop. 5]. Then $\operatorname{Spec} A$ is zero-dimensional and reduced and its underlying topological space is the one-point compactification of $k$ with its discrete topology. For every $\lambda \in k$, the corresponding quotient $A \rightarrow \kappa(\lambda)=k$ is a locally free and finitely generated $A$-module, hence projective. The direct sum $M=\oplus_{\lambda \in k} \kappa(\lambda)$ is a projective $A$-module with rank 1 over the open subset $k$ and rank 0 over its complement, which consists of a single point $\xi$.

The discrete additive group $G=(k,+)$ acts freely on $\operatorname{Spec} A$ and the quotient $X=\operatorname{Spec} A / G$ is an algebraic space consisting of two points $\{x, \xi\}$ where $x$ is open and $\xi$ is closed. Note that $X$ is not quasi-separated since the orbit of $x$ is not quasi-compact. The module $M$ descends to a locally projective $\mathcal{O}_{X}$-module $\mathcal{F}$ such that the rank over $x$ is one and the rank over $\xi$ is zero. The topological space $|X|$ is irreducible and hence the rank is not constant over irreducible components in the usual sense.

A flat module that has constant rank need not be so nice either as the following example shows.

\footnotetext{
${ }^{1}$ This condition is necessary with the naive notion of irreducible components, cf. Example (2.3).
} 
Example (2.4). If $M \subseteq \mathbb{Q}$ is the $\mathbb{Z}$-submodule generated by all $p^{-1}$, for prime numbers $p$, then $M$ is flat of constant rank 1 but neither projective nor finitely generated.

Proposition (2.5). Let $X$ be an algebraic stack and let $\mathcal{F}$ be a quasicoherent sheaf on $X$.

(i) If $X$ is an affine scheme, then $\mathcal{F}$ is locally projective if and only if $\mathcal{F}$ is projective.

(ii) If $X$ is a noetherian affine scheme and $\aleph \geq \aleph_{0}$ is an infinite cardinal, then $\mathcal{F}$ is projective with constant rank $\aleph$ if and only if $\mathcal{F}$ is free of rank $\aleph$.

(iii) If $X$ is noetherian, then $\mathcal{F}$ is locally projective of finite rank if and only if $\mathcal{F}$ is finitely generated and locally free.

(iv) If $X$ is noetherian, then $\mathcal{F}$ is locally projective if and only if $\mathcal{F}$ is locally free.

(v) If $X$ is a noetherian scheme, then $\mathcal{F}$ is locally free if and only if $\mathcal{F}$ is Zariski-locally free.

Proof. In each case, the "if" part is trivial. The necessity of the first condition follows from [RG71, I.3.1.4] (countable rank) or [RG71, II.2.5.1 and II.3.1.3] (general case). That conditions (ii) and (iii) are necessary is [Bas63, Cor. 3.2 \& Prop. 4.2] respectively. Since the rank of a locally projective sheaf is locally constant on a noetherian stack, the necessity of conditions (iv) and (v) follow from (i), (ii) and (iii),

Remark (2.6). Without the noetherian assumptions, statements (iii) and(iv) are false. If statement (ii) holds without the noetherian assumption, then so does (v). In particular, this would imply that on any stack $X$, a quasicoherent sheaf $\mathcal{F}$ is locally free if and only if $\mathcal{F}$ is locally projective, has locally constant rank and is finitely generated over the open locus of finite rank.

\section{RELAtive ASSASSins AND RELATIVE FAITHFulness}

In this section, we extend the notions of relative assassins [RG71, 3.2.2] and schematically dominant morphisms $\left.\mathrm{EGA}_{\mathrm{IV}}, 11.9-11.10\right]$ from schemes to algebraic stacks.

(3.1) Associated points - There is a unique notion of associated points of coherent sheaves on locally noetherian algebraic stacks such that

(i) it coincides with the usual one for schemes; and

(ii) if $f: X \rightarrow Y$ is a flat morphism between locally noetherian stacks and $\mathcal{F}$ is a coherent $\mathcal{O}_{Y}$-module, then $f\left(\operatorname{Ass}_{X}\left(f^{*} \mathcal{F}\right)\right) \subseteq \operatorname{Ass}_{Y}(\mathcal{F})$ with equality if $f$ is surjective.

The usual assassin satisfies (ii) for morphisms between schemes. Indeed, more precisely we have that

$$
\operatorname{Ass}_{X}\left(f^{*} \mathcal{F}\right)=\bigcup_{y \in \operatorname{Ass}_{Y}(\mathcal{F})} \operatorname{Ass}_{X_{y}}\left(\mathcal{O}_{X_{y}}\right)
$$

for any flat morphism $f: X \rightarrow Y$ between locally noetherian schemes EGA $_{I V}$, Prop. 3.3.1]. We may thus simply define $\operatorname{Ass}_{X}(\mathcal{F})$ for a coherent sheaf $\mathcal{F}$ 
on $X$ as $\operatorname{Ass}_{X}(\mathcal{F}):=p\left(\operatorname{Ass}_{U}\left(p^{*} \mathcal{F}\right)\right)$ where $p: U \rightarrow X$ is a presentation. One can also give a more intrinsic definition, cf. [Lie07, 2.2.6.3-2.2.6.7]. We abbreviate $\operatorname{Ass}(X)=\operatorname{Ass}_{X}\left(\mathcal{O}_{X}\right)$.

In particular, if $f: X \rightarrow Y$ is locally of finite type and $\xi \in|Y|$ is a point, then we may define $\operatorname{Ass}\left(X_{\xi}\right) \subseteq|f|^{-1}(\xi)$ as the image of $\operatorname{Ass}\left(X_{y}\right) \rightarrow|X|$ for any representative $y: \operatorname{Spec} k \rightarrow Y$ of $\xi$.

Definition (3.2) ([RG71, Déf. 3.2.2]). Let $f: X \rightarrow Y$ be a morphism of algebraic stacks that is locally of finite type. The relative assassin $\operatorname{Ass}(X / Y)$ is the subset $\bigcup_{y \in|Y|} \operatorname{Ass}\left(X_{y}\right)$ of $|X|$.

Note that $X$ and $Y$ need not be noetherian in the definition above, but the finiteness condition ensures that the fibers are locally noetherian. If $f$ is flat and $X$ and $Y$ are locally noetherian, then $\operatorname{Ass}(X)=\bigcup_{y \in \operatorname{Ass}(Y)} \operatorname{Ass}\left(X_{y}\right) \subseteq$ $\operatorname{Ass}(X / Y)$ by (3.1.1). The advantage of $\operatorname{Ass}(X / Y)$ is that it behaves well with respect to any base change $Y^{\prime} \rightarrow Y$, whereas $\operatorname{Ass}(X)$ does not behave well with respect to non-flat base change, e.g., passage to a fiber.

If $p: X^{\prime} \rightarrow X$ is flat and locally of finite type, then $p\left(\operatorname{Ass}\left(X^{\prime} / Y\right)\right) \subseteq$ $\operatorname{Ass}(X / Y)$ with equality if $p$ is surjective; this follows from property (ii) above.

Definition (3.3). Let $f: X \rightarrow Y$ be a morphism of algebraic stacks. We say that $f$ is schematically dominant if $\mathcal{O}_{Y} \rightarrow f_{*} \mathcal{O}_{X}$ is injective as a morphism of lisse-étale sheaves.

This agrees with the usual definition for schemes [EGA $\left.\mathrm{EV}_{\mathrm{IV}}, 11.10 .2\right]$ since that notion is stable under base change by flat morphisms that are locally of finite presentation $\mathrm{EGA}_{\mathrm{IV}}, 11.10 .5$ (ii) b)]. It follows that our notion for algebraic stacks also is stable under base change by flat morphisms that are locally of finite presentation. When $f$ is quasi-compact, the notion is stable under arbitrary flat base change [EGA

If $p: X^{\prime} \rightarrow X$ is another morphism and $f \circ p$ is schematically dominant, then so is $f$. If $f$ and $p$ are schematically dominant, then so is $f \circ p$. In particular, morphisms that are covering in the fppf topology are schematically dominant.

Definition (3.4). Let $S$ be an algebraic stack and let $f: X \rightarrow Y$ be a morphism of algebraic stacks over $S$. We say that $f$ is $S$-universally schematically dominant if $f^{\prime}: X \times{ }_{S} S^{\prime} \rightarrow Y \times{ }_{S} S^{\prime}$ is schematically dominant for every morphism $S^{\prime} \rightarrow S$.

Proposition (3.5). Let $S, X$ and $Y$ be algebraic stacks and let $f: X \rightarrow Y$ and $Y \rightarrow S$ be flat morphisms that are locally of finite presentation. The following are equivalent.

(i) The morphism $f$ is $S$-universally schematically dominant.

(ii) The image $f(X)$ contains the relative assassin $\operatorname{Ass}(Y / S)$.

Proof. Since $f$ is open and faithfully flat onto its image, we may assume that $f$ is an open immersion. As the question is fppf-local on $Y$ and $S$ we may assume that $Y$ and $S$ are affine schemes. The result is then $\mathrm{EGA}_{\mathrm{IV}}$, Prop. 11.10.10] (or [RG71, Cor. 3.2.6]). 
Definition (3.6). Let $f: X \rightarrow Y$ and $g: Y \rightarrow S$ be morphisms, locally of finite presentation, between algebraic stacks such that $g$ is flat. We say that $f$ is $S$-faithfully flat if $f$ is flat and the equivalent conditions of Proposition (3.5) hold.

This terminology is explained by the following lemma.

Lemma (3.7). Let $f: X \rightarrow Y$ and $\pi: Y \rightarrow S$ be morphisms of algebraic stacks. Assume that $f$ is $S$-universally schematically dominant. Given $\mathcal{F} \in$ $\mathbf{Q} \operatorname{Coh}(Y)$ and $\mathcal{G} \in \mathbf{Q} \operatorname{Coh}(S)$, we have that

(i) the unit map $\eta_{\pi^{*} \mathcal{G}}: \pi^{*} \mathcal{G} \rightarrow f_{*} f^{*} \pi^{*} \mathcal{G}$ is injective; and

(ii) a morphism $\theta: \mathcal{F} \rightarrow \pi^{*} \mathcal{G}$ is zero if and only if $f^{*} \theta$ is zero.

Proof. Consider $S^{\prime}=\operatorname{Spec}\left(\mathcal{O}_{S} \oplus \mathcal{G}\right)$, where $\mathcal{G}$ is square-zero, and let $X^{\prime}=$ $X \times_{S} S^{\prime}$ and $Y^{\prime}=Y \times_{S} S^{\prime}$. Then $f^{\prime}: X^{\prime} \rightarrow Y^{\prime}$ is schematically dominant, that is, $\mathcal{O}_{Y} \oplus \pi^{*} \mathcal{G} \rightarrow f_{*}\left(\mathcal{O}_{X} \oplus f^{*} \pi^{*} \mathcal{G}\right)$ is injective. It follows that $\eta_{\pi^{*} \mathcal{G}}$ is injective.

If $\theta$ is zero, then so is $f^{*} \theta$. Conversely, if $f^{*} \theta$ is zero, then so is $\eta_{\pi^{*} \mathcal{G}} \circ \theta=$ $\left(f_{*} f^{*} \theta\right) \circ \eta_{\mathcal{F}}$. It follows that $\theta$ is zero since $\eta_{\pi^{*} \mathcal{G}}$ is injective.

Lemma (3.8). Let $f: X \rightarrow Y$ and $\pi: Y \rightarrow S$ be flat morphisms, that are locally of finite presentation, between algebraic stacks. Let $\mathcal{F}_{0} \subseteq \mathcal{F}$ be quasi-coherent $\mathcal{O}_{S}$-modules and let $\mathcal{G}_{0} \subseteq \pi^{*} \mathcal{F}$ be a quasi-coherent $\mathcal{O}_{Y^{-}}$ submodule. Assume that $f$ is $S$-faithfully flat. Then $\mathcal{G}_{0} \subseteq \pi^{*} \mathcal{F}_{0}$ if and only if $f^{*} \mathcal{G}_{0} \subseteq f^{*} \pi^{*} \mathcal{F}_{0}$.

Proof. Let $\mathcal{F}^{\prime}=\mathcal{F} / \mathcal{F}_{0}$. Consider the map $\theta: \mathcal{G}_{0} \hookrightarrow \pi^{*} \mathcal{F} \rightarrow \pi^{*} \mathcal{F}^{\prime}$. Then $\mathcal{G}_{0} \subseteq \pi^{*} \mathcal{F}_{0}$ if and only if $\theta=0$ and $f^{*} \mathcal{G}_{0} \subseteq f^{*} \pi^{*} \mathcal{F}_{0}$ if and only if $f^{*} \theta=0$. Thus, the result follows from the previous lemma.

\section{Pure morphisms of Algebraic stacks}

We begin by recalling the definition of pure morphisms of schemes RG71, Déf. 3.3.3].

Definition (4.1). Let $f: X \rightarrow S$ be a morphism of schemes, locally of finite type. Let $s \in S$ be a point and let $(\widetilde{S}, \widetilde{s}) \rightarrow(S, s)$ be the henselization and $\widetilde{X}=X \times_{S} \widetilde{S}$. We say that $f$ is

(i) pure along $X_{s}$ if for every point $s_{1} \in \widetilde{S}$, every associated point $x_{1} \in \operatorname{Ass}\left(\widetilde{X}_{s_{1}}\right)$ is the generization of a point in $X_{s}$;

(ii) pure if $f$ is pure along $X_{s}$ for every $s \in S$; and

(iii) universally pure, if $f^{\prime}: X \times{ }_{S} S^{\prime} \rightarrow S^{\prime}$ is pure for every morphism $S^{\prime} \rightarrow S$.

(4.2) Examples - The two key examples of pure morphisms are RG71, Ex. I.3.3.4]:

(i) proper morphisms, and

(ii) faithfully flat morphisms, locally of finite type, with fibers that are geometrically irreducible without embedded components.

(4.3) Base change: descent - If $S^{\prime} \rightarrow S$ is faithfully flat and $f^{\prime}$ is pure, then $f$ is pure. Indeed, for every $s^{\prime} \in\left|S^{\prime}\right|$ with image $s \in|S|$, the morphism 
between henselizations $\left(\widetilde{S}^{\prime}, s^{\prime}\right) \rightarrow(\widetilde{S}, s)$ is surjective. If $x_{1} \in \operatorname{Ass}\left(\widetilde{X}_{s_{1}}\right)$, then there exists $x_{1}^{\prime} \in \operatorname{Ass}\left(\widetilde{X}_{s_{1}^{\prime}}^{\prime}\right)$ above $x_{1}$ (3.1) (ii) and, by purity, a specialization $x^{\prime} \in X_{s^{\prime}}^{\prime}$. Its image $x \in X_{s}$, is a specialization of $x_{1}$.

(4.4) Base change: stability - If $f$ is flat, pure and of finite presentation, then $f$ is universally pure [RG71, 3.3.7]. Also, every pure morphism of finite presentation is universally pure when $S$ is locally noetherian [SP, 05J8] but not for general $S[\mathrm{SP}, 05 \mathrm{JJ}]$.

(4.5) Composition - Let $f: X \rightarrow Y$ and $g: Y \rightarrow S$ be morphisms of schemes, locally of finite type. If $f$ and $g$ are pure, then $g \circ f$ need not be pure, e.g., the composition $\operatorname{Spec}(k[x, y] / x y-1) \hookrightarrow \operatorname{Spec} k[x, y] \rightarrow \operatorname{Spec} k[x]$ is not pure. On the other hand, if $f$ is flat and pure and $g$ is pure, then $g \circ f$ is pure. Indeed, the map $\tilde{f}: \widetilde{X}=X \times_{S} \widetilde{S} \rightarrow \widetilde{Y}=Y \times_{S} \widetilde{S}$ is pure along $X_{y}$ for every $y \in Y_{s}$ since the henselization of $Y$ at any point of $Y_{s}$ factors through $\tilde{Y}$. Moreover, since $\tilde{f}$ is flat, we have that $\tilde{f}\left(\operatorname{Ass}\left(\widetilde{X}_{s_{1}}\right)\right) \subseteq \operatorname{Ass}\left(\widetilde{Y}_{s_{1}}\right)$ for every $s_{1} \in \widetilde{S}$. Also, if $f$ is faithfully flat and $g \circ f$ is pure, then $g$ is pure. Indeed, for every point $s_{1} \in \widetilde{S}$, we have that $\tilde{f}\left(\operatorname{Ass}\left(\widetilde{X}_{s_{1}}\right)\right)=\operatorname{Ass}\left(\widetilde{Y}_{s_{1}}\right)$.

To extend purity to morphisms of stacks, we give a slightly different definition.

Definition (4.6). Let $f: X \rightarrow S$ be a morphism between algebraic stacks that is quasi-separated and locally of finite type. When $S$ is quasi-separated, we say that $f$ is weakly closed if $f(Z)$ is closed for every closed irreducible subset $Z \subset|X|$, such that the generic point of $Z$ is associated in its fiber. We say that $f$ is universally weakly closed, if $f^{\prime}: X \times_{S} S^{\prime} \rightarrow S^{\prime}$ is weakly closed for every morphism $S^{\prime} \rightarrow S$ where $S^{\prime}$ is quasi-separated.

The remarks in (4.2), (4.3) and (4.5) hold for "pure" replaced by "weakly closed". For Remark (4.3), note that $f$ is weakly closed if and only if $f(\overline{\{z\}})$ is stable under specialization for every $z \in \operatorname{Ass}(X / S)$, and this can be checked flat-locally on $S$. The analogue of Remark (4.4) is false, which is not surprising: the good notion is universally weakly closed for which we have the following valuative criterion.

Proposition (4.7). Let $f: X \rightarrow S$ be a quasi-separated morphism, locally of finite type, between algebraic stacks. Then the following are equivalent:

(i) $f$ is universally weakly closed;

(ii) for every valuation ring $V$ and morphism $\operatorname{Spec} V \rightarrow S$, the base change $X \times{ }_{S}$ Spec $V \rightarrow \operatorname{Spec} V$ is weakly closed; and

(iii) for every valuation ring $V$, morphism $\operatorname{Spec} V \rightarrow S$, and associated point $z$ in the generic fiber $X \times{ }_{S}$ Spec $K(V)$, the closure of $z$ in $\left|X \times_{S} \operatorname{Spec} V\right|$ surjects onto $\operatorname{Spec} V$.

If $f$ is a morphism of schemes, then this is equivalent to:

$\left(\mathrm{i}^{\prime}\right) f$ is universally pure.

Proof. Clearly, (i) $\Longrightarrow$ (ii) $\Longrightarrow$ (iii). If $f$ is a morphism of schemes, then trivially (i) $\Longrightarrow\left(\mathrm{i}^{\prime}\right)$ and we note that $\left(\mathrm{i}^{\prime}\right) \Longrightarrow$ (ii) since it is enough to verify (ii) for henselian valuation rings. 
To see that (iii) $\Longrightarrow$ (i) it is enough to prove that $f$ is weakly closed. Let $z \in|X|$ be a point that is associated in its fiber and let $Z=\overline{\{z\}}$. It is enough to prove that $f(Z)=\overline{\{f(z)\}}$. This can be verified after the base change $S^{\prime}=\operatorname{Spec} V \rightarrow S$ for every valuation ring $V$ and every dominant morphism Spec $V \rightarrow \overline{\{f(z)\}}$. Then $f(Z)=\operatorname{Spec} V$ by (iii) and the result follows.

Definition (4.8). Let $f: X \rightarrow Y$ be a flat morphism of finite presentation between algebraic stacks. We say that $f$ is pure if it is universally weakly closed.

This definition coincides with the usual definition for flat morphisms of schemes by (4.4). It also coincides with the definition of pure in Rom11, B.1].

The following lemma, which is a direct transcription of an argument in [RG71, proof of Prop. 3.3.6], shows that a flat morphism $X \rightarrow S$ of finite presentation is weakly closed if and only if the map $\operatorname{Ass}(X / S) \rightarrow S$ is closed under specializations, i.e., if subsets closed under specialization in $\operatorname{Ass}(X / S)$ maps to subsets closed under specialization in $S$.

Lemma (4.9). Let $S$ be a scheme and let $X$ be an algebraic stack that is flat and of finite presentation over $S$. Let $s, s_{1} \in|S|$ and $x_{1} \in \operatorname{Ass}\left(X_{s_{1}}\right)$. If $\left|X_{s}\right| \cap \overline{\left\{x_{1}\right\}} \neq \emptyset$, then $\operatorname{Ass}\left(X_{s}\right) \cap \overline{\left\{x_{1}\right\}} \neq \emptyset$.

Proof. We may assume that $S=\operatorname{Spec} A$ is affine. Pick a smooth presentation $p: U=\operatorname{Spec} B \rightarrow X$. If $\left|X_{s}\right| \cap \overline{\left\{x_{1}\right\}} \neq \emptyset$, then there exists a point $u_{1} \in U$ above $x_{1}$ such that $\left|U_{s}\right| \cap \overline{\left\{u_{1}\right\}} \neq \emptyset$. We may assume that $u_{1}$ is maximal in $p^{-1}\left(x_{1}\right)$ and then $u_{1} \in \operatorname{Ass}\left(U_{s_{1}}\right)$. Since $p\left(\operatorname{Ass}\left(U_{s}\right)\right)=\operatorname{Ass}\left(X_{s}\right)$, it is enough to prove that $\operatorname{Ass}\left(U_{s}\right) \cap \overline{\left\{u_{1}\right\}} \neq \emptyset$.

Let $u \in\left|U_{s}\right| \cap \overline{\left\{u_{1}\right\}}$ and let $\Sigma \subseteq \mathcal{O}_{U, u}$ be the set of elements whose images in $\mathcal{O}_{U, u} \otimes \kappa(s)$ are non-zero divisors. Then $\mathcal{O}_{U, u} \rightarrow \Sigma^{-1} \mathcal{O}_{U, u}$ is $A$-universally injective and $\Sigma^{-1} \mathcal{O}_{U, u}$ is a semi-local ring whose maximal ideals are associated points of $U_{s}$ [RG71, 3.2.5]. In particular, the morphism $\mathcal{O}_{U, u} \otimes \kappa\left(s_{1}\right) \rightarrow\left(\Sigma^{-1} \mathcal{O}_{U, u}\right) \otimes \kappa\left(s_{1}\right)$ is injective. Since $u_{1}$ is associated in $\operatorname{Spec}\left(\mathcal{O}_{U, u} \otimes \kappa\left(s_{1}\right)\right)$, this means that $u_{1} \in \operatorname{Spec}\left(\Sigma^{-1} \mathcal{O}_{U, u} \otimes \kappa\left(s_{1}\right)\right)$; hence $u_{1}$ is a generization of an associated point $u_{0}$ of $U_{s}$.

\section{HomologicAl PROJECTIVITY}

The main theorem of [RG71, §I.3] is the following relation between purity and projectivity for affine morphisms.

Theorem (5.1) (Raynaud-Gruson). Let $f: X \rightarrow Y$ be an affine finitely presented morphism of schemes. The following are equivalent:

(i) $f$ is flat and pure;

(ii) $f_{*} \mathcal{O}_{X}$ is locally projective; and

(iii) $f_{*} \mathcal{O}_{X}$ is locally free.

Proof. The equivalence between (i) and (ii) is [RG71, Thm. I.3.3.5]. The equivalence between (ii) and (iii) is [RG71, Cor. I.3.3.12]. Note that if $Y$ is noetherian, then the latter equivalence follows directly from Proposition (2.5) (iv). The non-noetherian case follows from the noetherian case 
using the equivalence between (i) and (ii) and using that pure morphisms behave well under approximation [RG71, Cor. I.3.3.10].

Local projectivity of $f_{*} \mathcal{O}_{X}$ is not local on $X$. To obtain a non-affine analogue of the theorem above, we introduce the following definition.

Definition (5.2). Let $f: X \rightarrow Y$ be a flat morphism of finite presentation between algebraic stacks. We say that $f$ is homologically projective (resp. strongly homologically projective) if there exists

(i) an fppf-covering $\left\{\operatorname{Spec}\left(A_{i}\right) \rightarrow Y\right\}$; and

(ii) flat morphisms $q_{i}: \operatorname{Spec}\left(B_{i}\right) \rightarrow X \times_{Y} \operatorname{Spec}\left(A_{i}\right)$, locally of finite presentation;

such that for every $i$

(a) the composition $\operatorname{Spec}\left(B_{i}\right) \stackrel{q_{i}}{\longrightarrow} X \times_{Y} \operatorname{Spec}\left(A_{i}\right) \rightarrow \operatorname{Spec}\left(A_{i}\right)$ makes $B_{i}$ into a projective $A_{i}$-module; and

(b) $q_{i}$ is $\operatorname{Spec}\left(A_{i}\right)$-faithfully flat (resp. faithfully flat), cf. Definition (3.6).

Here "homological" is to indicate that projective is interpreted as in homological algebra and not as in algebraic geometry. It should not be confused with the notion of cohomologically projective morphisms in Alp13, 3.18].

By definition, the notion of (strong) homological projectivity is stable under base change and fppf-local on the target. If $p: X^{\prime} \rightarrow X$ is faithfully flat and locally of finite presentation and $f \circ p$ is (strongly) homologically projective, then $f$ is (strongly) homologically projective but the converse does not hold. It is, a priori, not clear whether the composition of two (strongly) homologically projective morphisms is (strongly) homologically projective.

Recall that $X$ has the resolution property if every quasi-coherent sheaf of finite type on $X$ admits a surjection from a vector bundle.

Theorem (5.3). Let $f: X \rightarrow Y$ be a morphism of algebraic stacks that is flat and of finite presentation. Consider the following conditions:

(i) $f$ is affine and $f_{*} \mathcal{O}_{X}$ is locally projective;

(ii) $f$ is strongly homologically projective;

(iii) $f$ is homologically projective; and

(iv) $f$ is pure.

Then (i) $\Longrightarrow$ (ii) $\Longrightarrow$ (iii) $\Longleftrightarrow($ iv). If $f$ is affine, then all four conditions are equivalent. If $X$ has the resolution property fppf-locally on $Y$ (e.g., if $f$ is quasi-affine), then (ii) $\Longleftrightarrow$ (iii).

Proof. From the definitions, it follows that (i) $\Longrightarrow$ (ii) $\Longrightarrow$ (iii). To prove that (iii) $\Longrightarrow$ (iv), we may assume that $Y=\operatorname{Spec} A$ and that there is a $Y$-faithfully flat and finitely presented morphism $U=\operatorname{Spec} B \rightarrow X$ such that $B$ is a projective $A$-module. By Theorem (5.1), we have that $U \rightarrow Y$ is pure. Since the image of $U$ contains $\operatorname{Ass}(X / Y)$, it follows that $X \rightarrow Y$ is pure. When $f$ is affine, (iv) $\Longrightarrow$ (i) by Theorem (5.1).

For (iv) $\Longrightarrow$ (iii), suppose that $f$ is pure. As before we may assume that $Y$ is affine. Pick a smooth presentation $U=\operatorname{Spec} B \rightarrow X$. Let $y \in Y$ be a 
point. Then, by [RG71, Prop. 3.3.2], there exists a commutative diagram

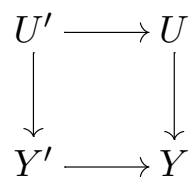

and a point $y^{\prime} \in Y^{\prime}$ above $y$ such that

- $U^{\prime} \rightarrow U$ and $Y^{\prime} \rightarrow Y$ are étale, and $\kappa(y)=\kappa\left(y^{\prime}\right)$;

- $U^{\prime}=\operatorname{Spec} B^{\prime}$ and $Y^{\prime}=\operatorname{Spec} A^{\prime}$ are affine and $B^{\prime}$ is a projective $A^{\prime}$-module; and

- the image of $U^{\prime} \rightarrow U$ contains $\operatorname{Ass}\left(U_{y}\right)$.

In particular, the image of $U^{\prime} \rightarrow U \rightarrow X$ contains $\operatorname{Ass}\left(X_{y}\right)$. After replacing $X, Y$ and $U$ by their pull-backs along the base change $Y^{\prime} \rightarrow Y$, we may assume that $Y^{\prime}=Y$.

We now claim that the image of $U^{\prime} \rightarrow U \rightarrow X$ contains $\operatorname{Ass}\left(X_{y_{1}}\right)$ for every generization $y_{1}$ of $y$. To see this, let $x_{1} \in \operatorname{Ass}\left(X_{y_{1}}\right)$. By the definition of purity, there exists a point $x \in X_{y} \cap \overline{\left\{x_{1}\right\}}$. By Lemma (4.9), there exists a point $x_{0} \in \operatorname{Ass}\left(X_{y}\right) \cap \overline{\left\{x_{1}\right\}}$. Since $x_{0}$ is in the image of $U^{\prime}$, so is its generization $x_{1}$.

By [RG71, Lem. 3.3.9], there is then an open neighborhood $y \in V \subseteq Y$ such that the image of $U^{\prime} \rightarrow U \rightarrow X$ contains $\operatorname{Ass}\left(X_{y_{1}}\right)$ for every $y_{1} \in V$. This means that $U^{\prime} \rightarrow U \rightarrow X$ is $Y$-faithfully flat over $V$, that is, $X \rightarrow Y$ is homologically projective over $V$. As the question is local on $Y$, it follows that $X \rightarrow Y$ is homologically projective.

Under the additional assumption on $X$, we will we prove that (iv) $\Longrightarrow$ (ii). For this, we may work locally on $Y$ and assume that $Y=\operatorname{Spec} A$ is affine and that $X$ has the resolution property. Then $X=\left[U / \mathrm{GL}_{n}\right]$ for some quasiaffine scheme $U$ [Tot04, Gro13]. By Jouanolou's trick, there is an affine vector bundle torsor $E \rightarrow U$ [Jou73, Lem. 1.5] (also see [Wei89, 4.3-4.4]). Since $E \rightarrow X$ is flat with geometrically integral fibers, hence flat and pure, it follows that $E \rightarrow X \rightarrow Y$ is pure (4.5). Since $E=\operatorname{Spec} B$ is affine, we have that $B$ is $A$-projective; thus, $f$ is strongly homologically projective.

\section{Existence of Minimal SUbSheaves}

Let $f: X \rightarrow Y$ be a faithfully flat morphism between quasi-compact algebraic stacks and let $\mathcal{F} \in \mathbf{Q} \operatorname{Coh}(Y)$. Assume that $\mathcal{G}_{0} \subseteq \mathcal{G}:=f^{*} \mathcal{F}$ is a quasi-coherent subsheaf of finite type. If $\mathcal{F}$ is the union of its quasi-coherent subsheaves $\mathcal{F}_{\lambda}$ of finite type, then, for sufficiently large $\lambda$, we have that $\mathcal{G}_{0} \subseteq f^{*} \mathcal{F}_{\lambda}$.

Conversely, if $\mathcal{G}=f^{*} \mathcal{F}$ is the union of its quasi-coherent subsheaves $\mathcal{G}_{\lambda}$ of finite type and for every $\mathcal{G}_{\lambda}$ there exists $\mathcal{F}_{\lambda} \subseteq \mathcal{F}$ of finite type such that $\mathcal{G}_{\lambda} \subseteq f^{*} \mathcal{F}_{\lambda}$, then $\mathcal{F}$ is the union of its subsheaves of finite type.

We will see that, under suitable hypotheses, for every $\mathcal{G}_{\lambda}$ of finite type as above there is a minimal $\mathcal{F}_{\lambda}$ as above and it is of finite type. This is, however, not always the case:

Example (6.1). Let $A$ be a discrete valuation ring with fraction field $K$ and uniformizing parameter $t$. Let $B=A \times K$, which is a faithfully flat 
$A$-algebra. Let $M=A$ and consider the submodule $N_{0}=(0 \times K) \subseteq$ $M \otimes_{A} B=B$. For every non-trivial ideal $M_{n}=\left(t^{n}\right) \subseteq A=M$, we then have that $N_{0} \subseteq M_{n} \otimes_{A} B=\left(t^{n}\right) \times K$. But the intersection is $\bigcap M_{n}=0$ and $N_{0} \nsubseteq\left(\bigcap M_{n}\right) \otimes_{A} B=0$. Hence, there is no minimal submodule $M^{\prime}$ of $M$ such that $N_{0} \subseteq M^{\prime} \otimes_{A} B$.

The problem in Example (6.1) is that infinite intersections do not commute with flat pull-back. This does not happen if we replace flatness with projectivity.

Lemma (6.2) (Serre). Let $A$ be a ring and let $B$ be an $A$-algebra which is projective as an $A$-module. Let $M$ be an $A$-module and let $N_{0} \subseteq M \otimes_{A} B$ be a $B$-submodule. Then there is a unique minimal $A$-submodule $M_{0} \subseteq M$ such that $N_{0} \subseteq M_{0} \otimes_{A} B$. Moreover,

(i) if $N_{0}$ is of finite type, then so is $M_{0}$; and

(ii) if $A^{\prime}$ is an A-algebra and we let $B^{\prime}=B \otimes_{A} A^{\prime}, M^{\prime}=M \otimes_{A} A^{\prime}, M_{0}^{\prime}:=$ $\operatorname{im}\left(M_{0} \otimes_{A} A^{\prime} \rightarrow M^{\prime}\right)$ and $N_{0}^{\prime}=\operatorname{im}\left(N_{0} \otimes_{B} B^{\prime} \rightarrow M^{\prime} \otimes_{A^{\prime}} B^{\prime}\right)$, then $M_{0}^{\prime}$ is the minimal $A^{\prime}$-submodule of $M^{\prime}$ such that $N_{0}^{\prime} \subseteq M_{0}^{\prime} \otimes_{A^{\prime}} B^{\prime}$.

Proof. Choose a free $A$-module $F$ such that $B$ is a direct summand of $F$ and pick a basis $\left\{e_{i}\right\}$ of $F$. Let $M_{0} \subseteq M$ be an $A$-submodule. Then $M_{0} \otimes_{A} B \subseteq$ $M_{0} \otimes_{A} F \subseteq M \otimes_{A} F$ and $M \otimes_{A} B \subseteq M \otimes_{A} F$. Let $x \in N_{0}$ be an element. Then $x=\sum_{i} x_{i} \otimes e_{i}$ in $M \otimes_{A} F$, and, using the retraction $F \rightarrow B$, we may also write $x=\sum_{i} x_{i} \otimes b_{i}$ in $M \otimes_{A} B$. Thus $x \in M_{0} \otimes_{A} B$ if and only if $x_{i} \in M_{0}$ for every $i$. It follows that the minimal submodule $M_{0}$ is the submodule generated by the $x_{i}$ 's when $x$ ranges over a set of generators of $N_{0}$. The remaining claims follows immediately from the construction of $M_{0}$.

Using purity, we give the following global version.

Theorem (6.3). Let $f: X \rightarrow Y$ be a flat morphism of finite presentation between algebraic stacks. Assume that $f$ is pure. Let $\mathcal{F} \in \mathbf{Q C o h}(Y)$ and let $\mathcal{G}_{0} \subseteq \mathcal{G}:=f^{*} \mathcal{F}$ be a quasi-coherent submodule. Then there is a unique minimal quasi-coherent submodule $\mathcal{F}_{0} \subseteq \mathcal{F}$ such that $\mathcal{G}_{0} \subseteq f^{*} \mathcal{F}_{0}$. Moreover,

(i) if $\mathcal{G}_{0}$ is of finite type, then so is $\mathcal{F}_{0}$; and

(ii) if $f^{\prime}: X^{\prime} \rightarrow Y^{\prime}$ is the base change of $f$ along a morphism $g: Y^{\prime} \rightarrow Y$, then the image $\mathcal{F}_{0}^{\prime}$ of $g^{*} \mathcal{F}_{0} \rightarrow g^{*} \mathcal{F}$ is the minimal quasi-coherent submodule such that $f^{\prime *} \mathcal{F}_{0}^{\prime}$ contains the image of $g^{\prime *} \mathcal{G}_{0} \rightarrow g^{\prime *} \mathcal{G}$.

Proof. By Theorem (5.3), $f$ is homologically projective. By fppf descent, it is enough to prove the statement after replacing $Y$ with an fppf cover. We may thus assume that $Y=\operatorname{Spec} A$ and that there exists a $Y$-faithfully flat morphism $q: X^{\prime}=\operatorname{Spec} B \rightarrow X$ of finite presentation such that $B$ is a projective $A$-module. If $\mathcal{F}_{0} \subseteq \mathcal{F}$ is a submodule, then $\mathcal{G}_{0} \subseteq f^{*} \mathcal{F}_{0}$ if and only if $q^{*} \mathcal{G}_{0} \subseteq q^{*} f^{*} \mathcal{F}_{0}$ (Lemma 3.8). We may thus replace $X$ with $X^{\prime}$ and assume that $X$ and $Y$ are affine. The theorem is then Lemma (6.2).

\section{Approximation of QUASI-COHerent Sheaves}

Let $X$ be a quasi-compact and quasi-separated algebraic stack. We recall that $X$ has the completeness property if every quasi-coherent $\mathcal{O}_{X}$-module is a directed colimit of finitely presented $\mathcal{O}_{X}$-modules and that $X$ has the partial 
completeness property if every quasi-coherent $\mathcal{O}_{X}$-module is the union of its finitely generated quasi-coherent submodules. In the terminology of Ryd15, $\S 4]$, these two conditions are the conditions $(\mathrm{C} 1)$ and $(\mathrm{C} 2)$ for the category $\mathrm{QCoh}(X)$ and they imply the corresponding facts for quasi-coherent $\mathcal{O}_{X^{-}}$ algebras.

We also make the following definition that extends Ryd15, Def. 4.7].

Definition (7.1). An algebraic stack $X$ is semi-noetherian (resp. pseudonoetherian) if it is quasi-compact, quasi-separated and $X^{\prime}$ has the partial completeness property (resp. completeness property) for every finitely presented morphism $X^{\prime} \rightarrow X$ of algebraic stacks.

Every pseudo-noetherian algebraic stack is semi-noetherian. Noetherian algebraic stacks, quasi-compact and quasi-separated schemes, algebraic spaces and Deligne-Mumford stacks are examples of pseudo-noetherian algebraic stacks [Ryd15, Thm. A].

Proposition (7.2). Let $f: X \rightarrow Y$ be a faithfully flat and pure morphism of finite presentation between quasi-compact and quasi-separated algebraic stacks. If $X$ has the partial completeness property, then so has $Y$. In particular, $X$ is semi-noetherian if and only if $Y$ is semi-noetherian.

Proof. Let $\mathcal{F} \in \mathbf{Q} \operatorname{Coh}(Y)$ and write $f^{*} \mathcal{F}$ as a union $\bigcup \mathcal{G}_{\lambda}$ of quasi-coherent submodules of finite type. By Theorem (6.3), for every $\lambda$ there exists a minimal quasi-coherent subsheaf $\mathcal{F}_{\lambda} \subseteq \mathcal{F}$ of finite type such that $\mathcal{G}_{\lambda} \subseteq f^{*} \mathcal{F}_{\lambda}$. If we let $\mathcal{F}^{\prime}=\bigcup \mathcal{F}_{\lambda} \subseteq \mathcal{F}$, then $f^{*} \mathcal{F}^{\prime}$ contains every $\mathcal{G}_{\lambda}$. It follows that $f^{*} \mathcal{F}^{\prime}=f^{*} \mathcal{F}$ and thus $\mathcal{F}^{\prime}=\mathcal{F}$ since $f$ is faithfully flat. We conclude that $Y$ has the partial completeness property.

Proposition (7.3). Let $X$ be an algebraic stack and let $p: X^{\prime} \rightarrow X$ be étale, representable, surjective and of finite presentation. Then $X$ is seminoetherian if and only if $X^{\prime}$ is semi-noetherian.

Proof. This is proven exactly as [Ryd15, Prop. 4.11]: étale dévissage [Ryd11, Thm. D] is used to reduce the question to where $p$ is either finite, surjective and étale or an étale neighborhood. These two cases follow from simplified versions of Ryd15, Lem. 4.9 and 4.10] where "completeness property" is replaced with "partial completeness property".

The main theorem will follow from the previous two propositions together with the following factorization result. For our main theorem we will only apply it to a smooth and representable morphism (the presentation of a stack).

Theorem (7.4) ([LMB00, 6.8], Rom11]). Let $f: X \rightarrow Y$ be a faithfully flat morphism of finite presentation with geometrically reduced fibers (e.g., $f$ smooth) between algebraic stacks. Then there exists an open substack $U \subseteq X$ and a factorization $\left.f\right|_{U}=h \circ g$ such that

(i) $g$ and $h$ are faithfully flat of finite presentation;

(ii) $h$ is representable and étale; and

(iii) $g$ has geometrically integral fibers. 
In particular, $g$ is pure. If $f$ is smooth, then $g$ is smooth and we can take $U=X$.

Proof. First assume that $f$ is smooth. Consider the connected factorization $X \rightarrow \pi_{0}(X / Y) \rightarrow Y$, which is described for morphisms of schemes in [LMB00, 6.8] and for an algebraic stack over an algebraic space in [Rom11, Thm. 2.5.2]. Since the construction commutes with base change, it generalizes to our situation as well. In this factorization $g: X \rightarrow \pi_{0}(X / Y)$ is smooth with geometrically connected fibers and $h: \pi_{0}(X / Y) \rightarrow Y$ is étale, representable and of finite presentation, but not necessarily separated Rom11, Thm. 2.5.2 (i), (ii)].

In the general case we use the functor of irreducible components of Romagny. The unicomponent locus $U \subseteq X$ is the subset of points that belong to exactly one irreducible component of their fibers. It is open and quasicompact and there is a factorization $U \rightarrow \operatorname{Irr}(X / Y) \rightarrow Y$ where the first morphism has geometrically integral fibers and the second is surjective, étale, representable and of finite presentation [Rom11, Thm. 2.5.2 (i), (iii)].

We now obtain the following equivalent form of the main theorem.

Theorem (7.5). Let $X$ be a quasi-compact and quasi-separated algebraic stack. Then $X$ is semi-noetherian.

Proof. Pick a smooth presentation $\operatorname{Spec} B \rightarrow X$. Theorem (7.4) gives a factorization Spec $B \rightarrow W \rightarrow X$ where $\operatorname{Spec} B \rightarrow W$ is smooth, surjective and pure and $W \rightarrow X$ is étale, surjective and of finite presentation. The result now follows from Propositions (7.2) and (7.3).

Remark (7.6). To answer Conjectures $\mathrm{A}$ and B, we may argue as in the proof of Theorem (7.5) using [Ryd15, Prop. 4.11 and Lem. 7.9]. This reduces the situation to where $X$ has a smooth presentation $U \rightarrow X$ with geometrically connected fibers. The author hopes that the purity of $U \rightarrow X$ and its characterization as homological projectivity can be used to settle the conjectures.

\section{Applications}

We conclude with some applications of the main theorem.

Theorem (8.1) (Zariski's main theorem). Let $f: X \rightarrow Y$ be a morphism between quasi-compact and quasi-separated algebraic stacks. Then the following are equivalent:

(i) $f$ is representable, separated and quasi-finite; and

(ii) there is a factorization $f=\bar{f} \circ j$ where $j$ is a quasi-compact open immersion and $\bar{f}$ is finite.

Proof. This follows from [LMB00, Thm. 16.5 (ii)] and the main theorem (taking into account that the finite presentation assumption of loc. cit. can be avoided by replacing the reference to [EGA] IV 8.12.6 with [EGA] IV 18.12.13). An essentially identical proof is given in Ryd15, Thm. 8.6 (ii)] (use the partial completeness property instead of the completeness property). 
Proposition (8.2). Let $X$ be a quasi-compact and quasi-separated algebraic stack and let $U \subseteq X$ be a quasi-compact open substack. Then there exists a closed immersion $Z \hookrightarrow X$ of finite presentation such that $U=X \backslash Z$.

Proof. Let $I \subseteq \mathcal{O}_{X}$ be the quasi-coherent sheaf of ideals defining $Z_{\text {red }}=$ $(X \backslash U)_{\text {red. }}$. Write $I=\bigcup I_{\lambda}$ as a union of quasi-coherent ideals of finite type. If $Z_{\lambda}$ denotes the finitely presented closed substack corresponding to $I_{\lambda}$, then $\cap Z_{\lambda}=Z_{\text {red }}$. Since $U$ is quasi-compact it follows that $\left|Z_{\lambda}\right|=\left|Z_{\text {red }}\right|$ for all sufficiently large $\lambda$. We may take $Z=Z_{\lambda}$ for any such $\lambda$.

As a third application, we have the existence of flattening stratifications for finitely presented morphisms.

Theorem (8.3). Let $X$ be a quasi-compact and quasi-separated algebraic stack and let $W \rightarrow X$ be a morphism of finite presentation. Then there exists a sequence of finitely presented closed substacks $\emptyset=X_{0} \hookrightarrow X_{1} \hookrightarrow \ldots \hookrightarrow X_{n}$ such that $\left|X_{n}\right|=|X|$ and the restriction of $W \rightarrow X$ to $X_{k} \backslash X_{k-1}$ is flat for every $k=1,2, \ldots, n$.

Proof. The result is well-known when $X$ is noetherian: let $X_{n}=X_{\text {red; }}$; pick a smooth presentation $p: \operatorname{Spec}(A) \rightarrow X_{n}$; choose a non-empty open subscheme $V \subseteq \operatorname{Spec}(A)$ over which $W$ is flat (generic flatness); let $X_{n-1}=$ $(X \backslash p(V))_{\text {red. }}$. The result now follows by noetherian induction.

If $X$ is affine, the result follows by standard limit methods: there is a noetherian affine scheme $X_{0}$, a morphism $X \rightarrow X_{0}$ and a morphism $W_{0} \rightarrow$ $X_{0}$ of finite presentation that pull-backs to $W \rightarrow X$. The pull-back of a solution to the problem for $W_{0} \rightarrow X_{0}$ gives a solution for $W \rightarrow X$.

In the general case, we pick a smooth presentation $p: X^{\prime}=\operatorname{Spec}(A) \rightarrow X$ and choose a filtration $X_{0}^{\prime} \hookrightarrow X_{1}^{\prime} \hookrightarrow \ldots \hookrightarrow X_{n}^{\prime}$ that solves the problem over $X^{\prime}$. We will prove that $X$ has a filtration of length $n$ that solves the problem. Set-theoretically, we will have $\left|X_{k}\right|=X \backslash p\left(X^{\prime} \backslash X_{k}^{\prime}\right)$. If $n=0$, the problem is trivial. By induction on $n$, we may assume that there exists a filtration of length $n-1$ on every closed substack $Q \hookrightarrow X$ such that $\left|p^{-1}(Q)\right| \subseteq\left|X_{n-1}^{\prime}\right|$.

The subset $p\left(X^{\prime} \backslash X_{n-1}^{\prime}\right)$ is open and quasi-compact, hence there is a finitely presented closed substack $Z \hookrightarrow X$ such that $X \backslash Z=p\left(X^{\prime} \backslash X_{n-1}^{\prime}\right)$ (Proposition 8.2).

Since $p$ is smooth, we have that $p^{-1}\left(X_{\text {red }}\right)=X_{\text {red }}^{\prime}$ and hence $p^{-1}\left(X_{\text {red }}\right) \hookrightarrow$ $X^{\prime}$ factors through $X_{n}^{\prime}$. Writing the nilradical of $\mathcal{O}_{X}$ as a union of quasicoherent ideals of finite type, we may write the nil-immersion $X_{\text {red }} \hookrightarrow X$ as an intersection of finitely presented nil-immersions $X_{\lambda} \hookrightarrow X$. For sufficiently large $\lambda$, we have that $p^{-1}\left(X_{\lambda}\right) \hookrightarrow X^{\prime}$ factors through $X_{n}^{\prime}$. Then $W \rightarrow X$ is flat over $X_{\lambda} \backslash Z$ for such $\lambda$ since $p^{-1}\left(X_{\lambda}\right) \backslash X_{n-1}^{\prime} \rightarrow X_{\lambda} \backslash Z$ is smooth and surjective.

We let $X_{n}=X_{\lambda}$ and $Q=Z \cap X_{\lambda}$. Then, by induction there is a filtration $X_{0} \hookrightarrow X_{1} \hookrightarrow \ldots \hookrightarrow X_{n-1} \hookrightarrow Q$ with $\left|X_{n-1}\right|=|Q|$ such that $W \rightarrow X$ is flat over the strata. The result follows.

As a fourth application, we have the existence of stratifications into gerbes for stacks with finitely presented inertia. 
Corollary (8.4). Let $X$ be a quasi-compact and quasi-separated algebraic stack with inertia of finite presentation. Then there exists a sequence of finitely presented closed substacks $\emptyset=X_{0} \hookrightarrow X_{1} \hookrightarrow \ldots \hookrightarrow X_{n}$ such that $\left|X_{n}\right|=|X|$ and $X_{k} \backslash X_{k-1}$ is an fppf gerbe over an affine scheme for every $k=1,2, \ldots, n$.

Proof. Apply Theorem (8.3) on $I_{X} \rightarrow X$ to obtain a stratification into fppf gerbes over quasi-compact and quasi-separated algebraic spaces. By Proposition (8.2), it remains to prove that a quasi-compact and quasi-separated algebraic space $S$ can be stratified into affine schemes. Pick an approximation $S \rightarrow S_{0} \rightarrow$ Spec $\mathbb{Z}$, that is, an algebraic space $S_{0}$ of finite presentation over Spec $\mathbb{Z}$ and an affine morphism $S \rightarrow S_{0}$ [Ryd15, Thm. D]. It is enough to stratify $S_{0}$ into affine schemes. This can be done by noetherian induction since $S_{0}$ has an open subspace that is a scheme.

For a general quasi-compact and quasi-separated algebraic stack, the inertia is only of finite type. In this case, it is not always possible to find finitely presented stratifications as in Corollary (8.4). In fact, sometimes even an infinite number of strata is required [SP, 06RE].

As a final application, we see that two different definitions of projectivity and quasi-projectivity over algebraic stacks are equivalent. Our main definition is analogous to that for schemes in EGA EGA E Défs. 5.3.1 and 5.5.2].

Definition (8.5). A representable morphism $f: X \rightarrow Y$ of algebraic stacks is

(i) quasi-projective if $f$ is of finite type and there exists an $f$-ample invertible $\mathcal{O}_{X}$-module; and

(ii) projective if $X$ is $Y$-isomorphic to a closed substack of a projective bundle $\mathbb{P}_{Y}(\mathcal{E})$ where $\mathcal{E}$ is a quasi-coherent $\mathcal{O}_{Y}$-module of finite type.

Note that being $f$-ample is an fppf-local property on the target $\mathrm{EGA}_{\mathrm{IV}}$, Cor. 2.7.2] and hence makes sense for representable morphisms. Similarly, projective bundles is a local construction on the base.

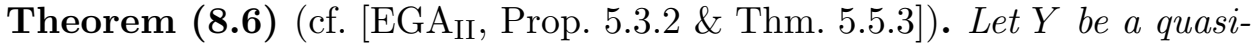
compact and quasi-separated algebraic stack and let $f: X \rightarrow Y$ be a representable morphism. Then

(i) $f$ is quasi-projective if and only if there exists a quasi-compact immersion $X \hookrightarrow \mathbb{P}_{Y}(\mathcal{E})$ over $Y$, where $\mathcal{E}$ is a quasi-coherent $\mathcal{O}_{Y^{-}}$ module of finite type.

(ii) $f$ is projective if and only if it is proper and quasi-projective.

Proof. If $i: X \rightarrow \mathbb{P}_{Y}(\mathcal{E})$ is a quasi-compact immersion, then $i^{*} \mathcal{O}_{\mathbb{P}(\mathcal{E})}(1)$ is very ample and $f$ is quasi-projective. Conversely, assume that $f$ is quasiprojective and let $\mathcal{L}$ be an $f$-ample invertible sheaf. There is a natural map $\sigma: f^{*} f_{*} \mathcal{L} \rightarrow \mathcal{L}$ and when this map is surjective, we have an induced morphism $r_{\mathcal{L}, \sigma}: X \rightarrow \mathbb{P}\left(f_{*} \mathcal{L}\right)$. Choose a presentation $g: Y^{\prime} \rightarrow Y$. After replacing $\mathcal{L}$ with a sufficiently large power, the invertible sheaf $g^{\prime *}(\mathcal{L})$ becomes very ample which implies that $\sigma$ is surjective and $r_{\mathcal{L}, \sigma}$ is an immersion $\mathrm{EGA}_{\mathrm{II}}$, Prop. 4.4.4]. Write $f_{*} \mathcal{L}$ as the union of its finitely generated submodules $\mathcal{E}_{\lambda}$. Then for sufficiently large $\lambda$, the map $\sigma_{\lambda}: f^{*} \mathcal{E}_{\lambda} \rightarrow \mathcal{L}$ is surjective and 
the induced morphism $r_{\mathcal{L}, \sigma_{\lambda}}: X \rightarrow \mathbb{P}\left(\mathcal{E}_{\lambda}\right)$ is an immersion EGA , pf. of Prop. 4.4.1 (ii)].

If $f$ is projective, then $f$ is quasi-projective (as before) and proper (check locally on $Y$ ). Conversely, if $f$ is quasi-projective and proper, then by (i)] there is an immersion $X \hookrightarrow \mathbb{P}_{Y}(\mathcal{E})$ which is closed since $f$ is proper.

\section{REFERENCES}

[Alp13] Jarod Alper, Good moduli spaces for Artin stacks, Ann. Inst. Fourier (Grenoble) 63 (2013), no. 6, 2349-2402.

[Bas63] Hyman Bass, Big projective modules are free, Illinois J. Math. 7 (1963), no. 1, $24-31$.

[EGA $\mathrm{I}$ ] A. Grothendieck, Éléments de géométrie algébrique. I. Le langage des schémas, Inst. Hautes Études Sci. Publ. Math. (1960), no. 4, 228.

[EGA $\mathrm{b}]$ A. Grothendieck, Éléments de géométrie algébrique. I. Le langage des schémas, second ed., Die Grundlehren der mathematischen Wissenschaften in Einzeldarstellungen, vol. 166, Springer-Verlag, Berlin, 1971.

[EGA $\mathrm{EII}_{\text {II }}$ A. Grothendieck, Éléments de géométrie algébrique. II. Étude globale élémentaire de quelques classes de morphismes, Inst. Hautes Études Sci. Publ. Math. (1961), no. 8, 222.

$\left[\mathrm{EGA}_{\mathrm{IV}}\right]$ A. Grothendieck, Éléments de géométrie algébrique. IV. Étude locale des schémas et des morphismes de schémas, Inst. Hautes Études Sci. Publ. Math. (1964$67)$, nos. 20, 24, 28, 32 .

[Gro13] Philipp Gross, Tensor generators on schemes and stacks, Preprint, Jun 2013, arXiv: 1306.5418

[HR14a] Jack Hall and David Rydh, Algebraic groups and compact generation of their derived categories of representations, Indiana Univ. Math. J. (2014), arXiv:1405.1890v2, accepted for publication.

[HR14b] Jack Hall and David Rydh, Coherent Tannaka duality and algebraicity of Homstacks, Preprint, May 2014, arXiv:1405.7680v2

[Jou73] J. P. Jouanolou, Une suite exacte de Mayer-Vietoris en K-théorie algébrique, Algebraic $K$-theory, I: Higher $K$-theories (Proc. Conf., Battelle Memorial Inst., Seattle, Wash., 1972), Springer, Berlin, 1973, pp. 293-316. Lecture Notes in Math., Vol. 341.

[Kap58] Irving Kaplansky, Projective modules, Ann. of Math (2) 68 (1958), no. 2, 372377.

[Lie07] Max Lieblich, Moduli of twisted sheaves, Duke Math. J. 138 (2007), no. 1, 23118.

[LMB00] Gérard Laumon and Laurent Moret-Bailly, Champs algébriques, Springer-Verlag, Berlin, 2000.

[Oli68] Jean-Pierre Olivier, Anneaux absolument plats universels et épimorphismes à buts réduit, Séminaire d'Algèbre Commutative dirigé par Pierre Samuel: 19671968. Les épimorphismes d'anneaux, Exp. No. 6, Secrétariat mathématique, Paris, 1968, p. 12.

[RG71] Michel Raynaud and Laurent Gruson, Critères de platitude et de projectivité. Techniques de "platification" d'un module, Invent. Math. 13 (1971), no. 1, 1-89.

[Rom11] Matthieu Romagny, Composantes connexes et irréductibles en familles, Manuscripta Math. 136 (2011), no. 1-2, 1-32.

[Ryd11] David Rydh, Étale dévissage, descent and pushouts of stacks, J. Algebra 331 (2011), 194-223.

[Ryd15] David Rydh, Noetherian approximation of algebraic spaces and stacks, J. Algebra 422 (2015), 105-147, arXiv:0904.0227v4.

[SGA 3 M. Demazure and A. Grothendieck (eds.), Schémas en groupes, Springer-Verlag, Berlin, 1970, Séminaire de Géométrie Algébrique du Bois Marie 1962/64 (SGA 3). Dirigé par M. Demazure et A. Grothendieck. Lecture Notes in Mathematics, Vol. 151-153. 
[SP] The Stacks Project Authors, Stacks project, http://stacks.math.columbia.edu/

[Tot04] Burt Totaro, The resolution property for schemes and stacks, J. Reine Angew. Math. 577 (2004), 1-22.

[Wei89] Charles A. Weibel, Homotopy algebraic $K$-theory, Algebraic $K$-theory and algebraic number theory (Honolulu, HI, 1987), Contemp. Math., vol. 83, Amer. Math. Soc., Providence, RI, 1989, pp. 461-488.

KTH Royal Institute of Technology, Department of Mathematics, SE-100 44 Stockholm, Sweden

E-mail address: dary@math.kth.se 